\title{
Osten holt auf, benötigt aber mehr Investitionen
}

\author{
Das dynamische Gleichgewicht, das sich in der deutschen Volkswirtschaft herausgebildet hat, ist \\ im produktiven Bereich überwiegend durch eine Annäherung der neuen Bundesländer an die alten \\ Bundesländer charakterisiert. Im Osten entwickelt sich die Produktivität besonders dynamisch und \\ fast ausschließlich aufgrund des technischen Fortschritts. Dieser Prozess könnte durch Anreize für \\ unternehmerische Investitionen beschleunigt werden. Angesichts der Größenordnung zusätzlich \\ zu investierender Beträge - es fehlen ca. 45 Mrd. Euro, um die Kapitalausstattung anzugleichen - \\ dürfte der Staat sehr schnell überfordert sein, wollte man ihm allein diese Aufgabe zuweisen.
}

Manche behaupten, dass „der Prozess der wirtschaftlichen Konvergenz zwischen Ost und West bereits vor rund 25 Jahren zum Erliegen"1 gekommen ist. Ein anderer Autor sieht entvölkerte Landstriche in den neuen Bundesländern, und behauptet, dass der Osten Deutschlands seine Wirtschaftskraft verloren habe. ${ }^{2}$ Als mögliche Erklärung für die schleppende Angleichung beispielsweise des Lohnniveaus wird spekuliert, dass ,in den frühen 1990er Jahren das durchschnittliche Bildungsniveau in Ostdeutschland vergleichsweise gering ausgefallen sei“"3 und sich dieser Unterschied im Humankapital verfestigt haben könnte. Demnach wären niedrigere Löhne dauerhaft hinzunehmen, also auf das politische Ziel der Konvergenz zu verzichten.

Abgesehen davon, dass die zuletzt genannte Hypothese auf einem inzwischen überwundenen Vor- und Fehlurteil beruht, ${ }^{4}$ liefert eine Studie des Deutschen Instituts für Wirtschaftsforschung (DIW) Anlass für eine tiefere Analyse, wonach beim Vergleich entwickelter Volkswirtschaften „kein Zusammenhang zwischen der Veränderung des Qualifikationsniveaus und der Produktivitätsentwicklung sichtbar

(C) Der/die Autor(en) 2020. Open Access: Dieser Artikel wird unter der Creative Commons Namensnennung 4.0 International Lizenz (https:// creativecommons.org/licenses/by/4.0/deed.de) veröffentlicht.

Open Access wird durch die ZBW - Leibniz-Informationszentrum Wirtschaft gefördert.

1 D. Marin: 30 Jahre Politikversagen: Deutschland mit zwei Seelen, in: Wirtschaftsdienst, 99. Jg. (2019), H. 10, S. 670 f., https://archiv.wirtschaftsdienst.eu/jahr/2019/10/30-jahre-politikversagen-deutschland-mit-zwei-seelen/ (28.11.2019).

2 G. Schnabl: Ostdeutschland: Leeres Land, in: Zeit, Nr. 40/2018, S. 27, https://www.zeit.de/2018/40/ostdeutschland-chemnitz-toleranz-wirtschaftskraft (19.11.2019).

3 V. Grossmann, T. Steger: Konvergieren die Einkommen zwischen Ostund Westdeutschland?, Beitrag zur „Ökonomenstimme“ vom 7.5.2013, https://www.oekonomenstimme.org/artikel/2013/05/konvergierendie-einkommen-zwischen-ost--und-westdeutschland/(8.1.2020).

4 Einen differenzierten und zugleich anschaulichen Einblick in Vor- und Nachteile der Schulsysteme beider deutscher Staaten findet man bei E. Urban, K. Pieper: Schulsystem in der DDR und heute: „Die Ostdeutschen lachen sich scheckig“, n-tv Beitrag vom 2.11.2018. ist" ${ }^{5}$ und folglich nach anderen Erklärungen für Produktivitätsunterschiede gesucht werden müsse. Als einen der wichtigeren Faktoren für die Entwicklung der Produktivität wird auf die Lohnentwicklung hingewiesen, die auch die Divergenzen zwischen der wirtschaftlichen Entwicklung in Ost und West zu einem Teil erklärt.

\section{Ein Maß für wirtschaftliche Konvergenz}

Das Bruttoinlandsprodukt (BIP) - weithin als das Maß für die Wirtschaftskraft eines Landes akzeptiert - belief sich 2018 auf 3386 Mrd. Euro für Gesamtdeutschland. Davon wurden Waren und Dienstleistungen im Wert von 2867 Mrd. Euro in den alten, und von 372 Mrd. Euro in den neuen Bundesländern produziert (jeweils ohne Berlin). ${ }^{6}$ Demnach leistet der Westen 7,7-mal mehr als der Osten - ein Verhältnis, das seit 1995 mit kleinen Schwankungen nahezu trendlos um die 8 pendelt. Niemand erwartet, dass die neuen Bundesländer diesen enormen Abstand jemals merklich reduzieren könnten - schließlich gab es 2018 in den alten Bundesländern 6,5-mal mehr Erwerbstätige (mit wachsender Tendenz seit 1995), die von einem 6,5-mal größeren Kapital unterstützt werden (Summe realer Nettoanlagevermögen, aktuellste Angabe 2016, seit 2010 steigt das Verhältnis zwischen West- und Ost-Kapital). Obwohl

5 K. Brenke: Produktivitätswachstum sinkt trotz steigendem Qualifikations niveau der Erwerbstätigen, in: DIW Wochenbericht, Nr. 33/2019, S. 578.

6 Volkswirtschaftliche Gesamtrechnungen der Länder: Bruttoinlandsprodukt, Bruttowertschöpfung in den Ländern der Bundesrepublik Deutschland 1991 bis 2018, Berechnungsstand August 2018/Februar 2019. Neben der Tabelle R1B1.xlsx sind für die Analyse auch die anderen Tabellen R1B2-B5 verwendet worden.

Doz. Dr. habil. Georg Quaas ist Hochschullehrer an der Universität Leipzig. 


\section{Abbildung 1}

\section{Arbeitsproduktivität: Kettenindex}

$2010=100$

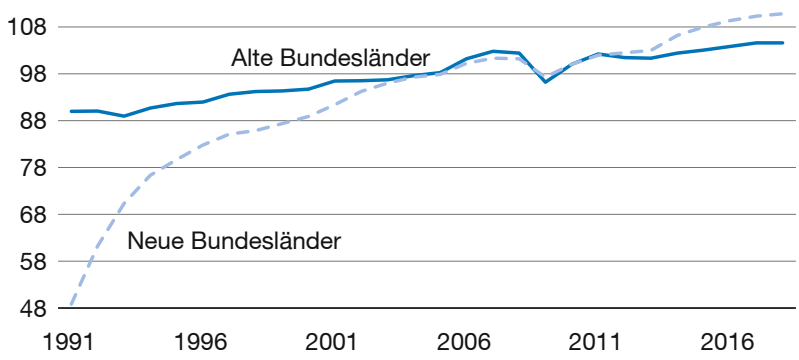

Quelle: Volkswirtschaftliche Gesamtrechnungen der Länder.

die produktiven Faktoren im Westen 6,5-mal stärker ausgeprägt sind, ist die Leistungskraft 7,7-mal höher. Das verweist auf bestehende Produktivitätsunterschiede, von denen man realistischerweise erwarten kann, dass sie im Laufe der Zeit abgebaut werden. Damit ist der Kern für einen volkswirtschaftlichen Ost-West-Vergleich benannt, und damit die Grundlage für die Beantwortung der Frage, ob ein Konvergenzprozess stattfindet oder ob dieser bereits zum Stillstand gekommen ist.

\section{Darstellung der Arbeitsproduktivität}

Für die Darstellung der Arbeitsproduktivität gibt es verschiedene Möglichkeiten, deren Grundlage jedoch einheitlich das reale BIP misst, das durch eine Arbeitseinheit durchschnittlich in einem bestimmten Gebiet und einem bestimmten Zeitabschnitt erzeugt wurde. Der Faktor Arbeit wird entweder durch die Erwerbstätigenzahl oder die Erwerbstätigenstunde definiert. In den letzten Jahren hat sich als Standard die Darstellung durch einen Kettenindex durchgesetzt, der den Vorteil hat, die Veränderung der zugrunde liegenden Größe kumulativ (multiplikativ) darzustellen. Abbildung 1 zeigt die Entwicklung der Arbeitsproduktivität in den alten und den neuen Bundesländern als Kettenindex. Dem liegt das preisbereinigte BIP je Erwerbstätigen zugrunde, das im jeweiligen Jahr erzeugt worden ist. Es wird deutlich, dass die Produktivität im Osten mit Ausnahme der Periode von 2002 bis 2010 deutlich schneller wächst als in den alten Bundesländern; insbesondere ist festzuhalten, dass der Konvergenzprozess ab 2011 weiter voranschreitet.

Die Darstellung mit einem Kettenindex hat den Nachteil, Niveauunterschiede zu nivellieren. Letztere werden in der Abbildung 2 auf der Grundlage zusätzlicher eigener Berechnungen gezeigt. Demnach haben die ostdeutschen Bundesländer noch einen weiten Weg vor sich, um auch nur in die Nähe der westdeutschen Produktivität zu gelangen. Obwohl der Verlauf der Kurven identisch ist, kann man
Abbildung 2

Arbeitsproduktivität pro Erwerbstätigen in 1000 Euro

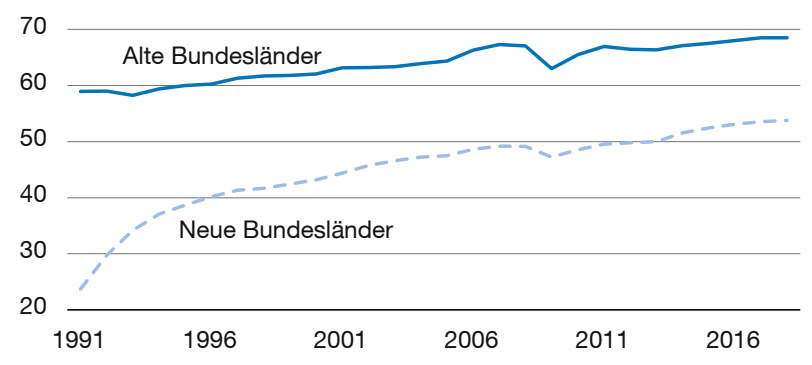

Quelle: Volkswirtschaftliche Gesamtrechnungen der Länder; eigene Rechnungen.

in der Abbildung 2 kaum noch erkennen, dass eine Konvergenz stattfindet. Das ist ein Nachteil der Darstellung der Arbeitsproduktivität in Euro je Erwerbstätigen und Jahr.

\section{Alternative Maße für die Produktivität}

In den Volkswirtschaftlichen Gesamtrechnungen (VGR) der Länder werden verschiedene Zeitreihen berichtet, die oberflächlich betrachtet alle als „Arbeitsproduktivität“ gedeutet werden könnten: das Bruttoinlandsprodukt (BIP) oder die Bruttowertschöpfung (BWS); diese gemessen in jeweiligen Preisen oder preisbereinigt; je Erwerbstätigen oder je Erwerbstätigenstunde und schließlich je Einwohner. Kombiniert man alle Alternativen miteinander, so ergeben sich zwölf verschiedene Variable, von denen aber "nur" zehn berichtet werden. Es fehlt die Variable „BWS je Einwohner“. Es darf vermutet werden, dass sie sich zwar berechnen lässt, aber ökonomisch nicht sinnvoll ist. In diesem Zusammenhang stellt sich die Frage, ob und wie die berichtete Größe „BIP je Einwohner" sinnvoll interpretiert werden kann.

Obwohl es in der Literatur zahlreiche Interpretationen gibt, die sogar das nominale BIP je Einwohner als Indikator für die wirtschaftliche Leistungskraft eines Landes definieren, wird hier an der internationalen Konvention festgehalten, dass es sich um ein - wenn auch grobes und konventionelles - Maß für den Wohlstand eines Landes handelt: „GDP per capita is a universal measure globally for gauging the prosperity of nations. "“7 Wenn der Wohlstand so wie die Produktivität in Realgrößen gemessen wird, unterscheiden sich beide nur durch den Faktor „Erwerbstätige je Einwohner", der ein Maß für den Beschäftigungsgrad ist. ${ }^{8}$ Die Abbildung 3 stellt die Entwicklung des Wohlstands

7 Per Capita GDP, in: Investopedia, https://www.investopedia.com/ terms/p/per-capita-gdp.asp (28.11.2019).

8 Nicht zu verwechseln mit der Beschäftigungsquote, bei der die Erwerbstätigenzahl auf die Zahl der Personen im erwerbsfähigen Alter (Erwerbspersonen) bezogen wird. 


\section{Abbildung 3}

Wohlstand: Bruttoinlandsprodukt pro Einwohner in 1000 Euro

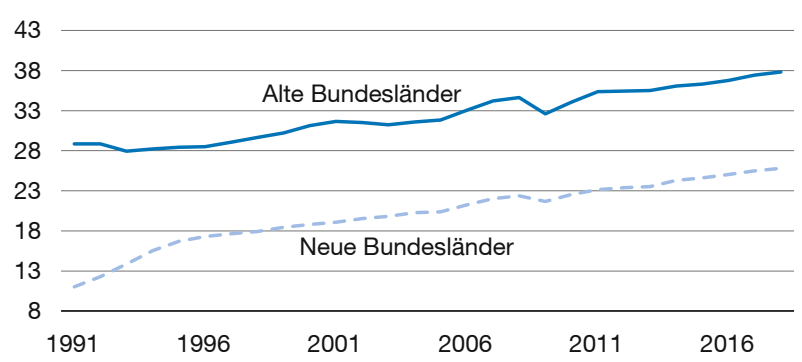

Quelle: Volkswirtschaftliche Gesamtrechnungen der Länder.

und die Abbildung 4 die des Beschäftigungsgrades differenziert für die alten und die neuen Bundesländer dar.

Um den Zusammenhang zu den ersten Abbildungen explizit herzustellen: Die Differenzen im Wohlstand (vgl. Abbildung 3) können komplett aus den Differenzen der Produktivität (vgl. Abbildung 2) und denen im Beschäftigungsgrad (vgl. Abbildung 4) erklärt werden. Der Zusammenhang wird komplizierter, wenn die Produktivität je Erwerbstätigenstunde angegeben wird, da die durchschnittlichen Arbeitszeiten in West und Ost verschieden sind.

\section{Beschäftigungsgrad}

Wie kommt es, dass der Beschäftigungsgrad in den neuen Bundesländern deutlich geringer ausfällt?

1. Pendler: Eine Berücksichtigung der Pendler zwischen Ost und West würde die Kurven zwar annähern, aber den Unterschied nicht beseitigen. Beispielsweise arbeiteten 414821 Ostdeutsche zum Stichdatum Ende Juni 2018 im Westen und 173544 Westdeutsche im Osten. ${ }^{9}$ Zieht man den Pendlersaldo von den Erwerbstätigen in den alten Bundesländern ab und addiert inn zu den neuen Bundesländern, ändert sich der Beschäftigungsgrad von $55,2 \%$ auf $54,8 \%$ im Westen, und von $47,9 \%$ auf $49,9 \%$ im Osten. Es verbleibt immer noch ein erheblicher Unterschied.

2. Wanderungen: Nach Angaben des Statistischen Bundesamtes und aufgrund eigener Berechnungen sind von 1991 bis 2018 ca. 1,2 Mio. mehr Menschen vom Osten in den Westen Deutschlands "gewandert"

9 Statistik der Bundesagentur für Arbeit: Arbeitsmarkt in Zahlen, Sozialversicherungspflichtig Beschäftigte - Pendler nach Ländern, Nürnberg, Stichtag 30.6.2018.

\section{Abbildung 4}

Beschäftigungsgrad: Erwerbstätige pro Einwohner in $\%$

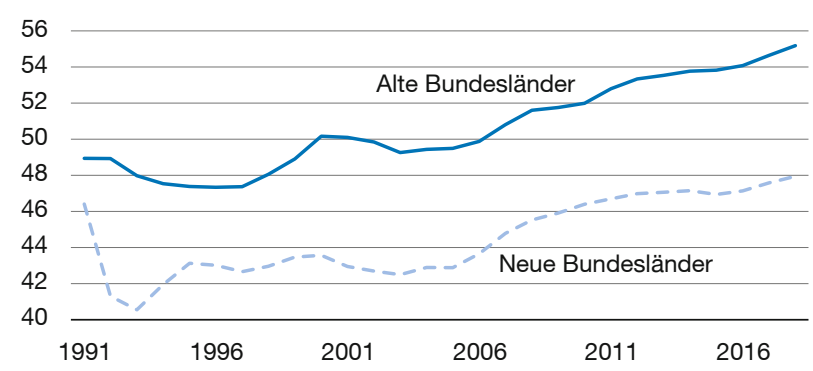

Quelle: Volkswirtschaftliche Gesamtrechnungen der Länder; eigene Rechnungen.

als umgekehrt. ${ }^{10}$ Berücksichtigt man außerdem die Altersstruktur, ${ }^{11}$ dürften von dieser Kohorte 2018 noch 1,06 Mio. Menschen erwerbstätig sein. Kontrafaktisch betrachtet lassen sich damit die Beschäftigungsgrade $54,1 \%$ (West) und 53,8\% (Ost) berechnen, die ohne Pendler und Abwanderungen bestehen würden. Man kann also davon ausgehen, dass die Situation, wie sie in Abbildung 4 dargestellt wird, im Wesentlichen durch den Sog zustande kommt, der vom westdeutschen Arbeitsmarkt ausgeht.

\section{Unterschiede bei den Arbeitsentgelten}

Ein Spruch auf den Montagsdemos ab November 1989 lautete: „Kommt die D-Mark, bleiben wir, kommt sie nicht, geh'n wir zu ihr." Inzwischen ist nicht mehr die generelle, sondern die konkrete Verfügbarkeit über eine harte Währung in Form von Löhnen und Gehältern das Problem der Ostdeutschen. Abbildung 5 zeigt einen nahezu konstanten Abstand zwischen West- und Ost-Arbeitsentgelten. Die Differenz ist in Abbildung 6 (rechte Skala, durchgehende Linie) dargestellt; sie bewegt sich schon seit geraumer Zeit zwischen 8000 und 9000 Euro pro Jahr. Die beiden Abbildungen machen deutlich, dass die Einschätzung der Konvergenz auch von der zugrunde liegenden Begrifflichkeit abhängt. Fokussiert man sich auf die Differenz, so dürfte die Behauptung einer Konvergenz Zweifel wecken. Betrachtet man dagegen die Relation zwischen beiden Kurven, so nimmt sie stetig ab (vgl. Abbildung 6, linke Skala, gepunktete Linie). Diese Annäherung kommt vor allem dadurch zustande, dass sich das Niveau beider Zeitreihen erhöht und damit inr Verhältnis zueinander kleiner wird.

10 Statistisches Bundesamt (Destatis) 2019: Wanderungssaldo OstWestwanderung für Ostdeutschland (ohne Berlin) je 1000 Einwohner. Die Rechnungen findet man unter http://www.georg-quaas.de/calculi.htm (8.1.2020).

11 Statistisches Bundesamt/Genesis-Online: Altersstruktur [der neuen Bundesländer] zum Stichtag 9.5.2011. 


\section{Abbildung 5}

\section{Arbeitsentgelte}

in 1000 Euro

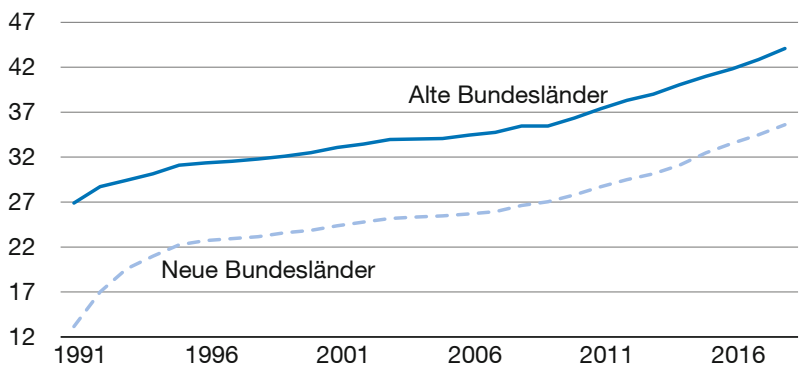

Quelle: Volkswirtschaftliche Gesamtrechnungen der Länder.

Es liegt auf der Hand, dass das Lohngefälle eine entscheidende Ursache für die Abwanderung und das Pendeln und damit für den geringeren Beschäftigungsgrad in den neuen Bundesländern ist. Die dortigen Veränderungen der Einwohnerzahl und der Erwerbstätigenzahl können ökonometrisch zu $70 \%$ bzw. $60 \%\left(\mathrm{R}^{2}\right)$ allein durch das Verhältnis der Arbeitsentgelte und seiner Veränderungen erklärt werden.

\section{Produktivitätsdifferenz}

Offen ist noch eine Erklärung der unterschiedlichen $\mathrm{Ni}$ veaus in der Arbeitsproduktivität. Zwar findet eine Konvergenz nach wie vor statt (vgl. Abbildung 1), aber es ist zweifelhaft, ob die Annäherung in absehbarer Zeit zu einer Parität führen wird (vgl. Abbildung 2). Wesentliche Faktoren zur Erklärung der Produktivität sind die Ausbildung der Arbeitskräfte und ihre Ausstattung mit Kapital.

\section{Humankapital}

Die These, dass die gut ausgebildeten Arbeitnehmer in den Westen pendeln oder auswandern, und die weniger qualifizierten im Osten verbleiben, ist wenig glaubhaft, wenn man sich vergleichend die Entwicklung des technologischen Fortschritts in Ost und West anschaut. Ein Maß dafür ist das Solow-Residuum. ${ }^{12}$ Abstrahiert man von der Umbruchzeit in den Jahren 1991 bis 1995, in denen dieses Maß besonders hoch war, und beginnt die Messung der totalen Faktorproduktivität 1995 mit dem Index 100, so ist sie in den neuen Bundesländern bis 2016 im Durchschnitt um 24\%, und in den alten um $13 \%$ gewachsen (vgl. Tabelle 1). Die Vertreter jener These müssen sich die Frage gefallen lassen, wie die neuste Technik von einer angeblich wenig qualifizierten Arbeitnehmerschaft gemeistert werden konnte.

12 Die hier angewandte Methodik wurde detailliert erläutert in G. Quaas: Test der neoklassischen Produktionsfunktion, MPRA-Paper, Nr. \#86368, 2018.

\section{Abbildung 6 \\ Vergleich westdeutscher mit ostdeutschen Arbeitsentgelten}

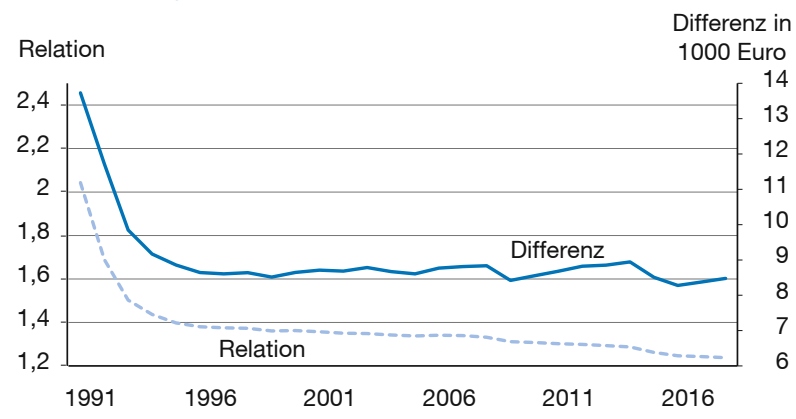

Quelle: Volkswirtschaftliche Gesamtrechnungen der Länder; eigene Rechnungen.

\section{Wirtschaftsstruktur}

Beliebt ist auch die These, dass die Unterschiede in der Produktivität auf regionale und sektorale Unterschiede zurückzuführen seien. So behaupten Belitz et al., dass „Ostdeutschland ... deutlich stärker ländlich geprägt [ist] als Westdeutschland."13 Falsch wäre es, aus dieser Feststellung und der Tatsache, dass die Aktivitäten des Sektors A (Land- und Forstwirtschaft, Fischerei) vorzugsweise im ländlichen Raum stattfinden, darauf zu schließen, dass die Wirtschaft in Ostdeutschland ländlich geprägt wäre. Für eine solche Charakteristik ist der Anteil der Land- und Forstwirte plus Fischer (im Weiteren kurz: „Landwirte") an der Gesamtzahl der Erwerbstätigen mit 2,0 \% im Jahr 2018 zu gering (vgl. Tabelle 1). Außerdem wird im Osten ein im Vergleich zum Westen größerer prozentualer Anteil der Bruttowertschöpfung der gewerblichen Wirtschaft in den ländlichen Regionen erwirtschaftet. ${ }^{14}$

Die von den Autoren behauptete stärkere ländliche Prägung besteht in einem Unterschied von 0,7 Prozentpunkten, das sind ca. 40000 Landwirte mehr als die westdeutsche "Benchmark" vorgibt. Hinzu kommt, dass die Landwirte der neuen Bundesländer, vermutlich dank der großzügigeren Parzellierung der Nutzfläche, die den Einsatz der modernsten Technik erlaubt, im sektoralen Produktivitätsvergleich mit den alten Bundesländern seit Jahren die Nase vorn haben - mit Ausnahme des extremen Jahres $2018 .{ }^{15}$ Die 40000 „überschüssigen“ ostdeutschen Er-

13 H. Belitz, A. Schiersch, T. Stühmeier: Produktivitätsentwicklung: Potenziale in Stadt und Land, in: Wirtschaftsdienst, 99. Jg. (2019), H. 5, S. 355, https://archiv.wirtschaftsdienst.eu/jahr/2019/5/produktivitaetsentwicklung-potenziale-in-stadt-und-land/ (28.11.2019).

14 Vgl. ebenda, S. 357.

15 Wird die Produktivität in Erwerbstätigenstunden gemessen, so liegen die neuen Bundesländer vorn, seitdem es Daten über das Arbeitsvolumen gibt. Siehe die Berechnung der verschiedenen Maße auf http:// www.georg-quaas.de/calculi.htm (8.1.2020). 
Tabelle 1

Volkswirtschaftliche Parameter

\begin{tabular}{|c|c|c|c|c|c|c|c|c|c|c|c|c|c|c|}
\hline \multirow[t]{2}{*}{ Jahr } & \multicolumn{2}{|c|}{$\begin{array}{l}\text { Totale Faktor- } \\
\text { produktivität }\end{array}$} & \multicolumn{2}{|c|}{$\begin{array}{c}\text { Nettoin- } \\
\text { vestitionsquote }\end{array}$} & \multicolumn{2}{|c|}{ Rendite } & \multicolumn{2}{|c|}{$\begin{array}{c}\text { Anteil der } \\
\text { Arbeitsentgelte }\end{array}$} & \multicolumn{2}{|c|}{$\begin{array}{l}\text { Land- } \\
\text { wirtschaft }\end{array}$} & \multicolumn{2}{|c|}{$\begin{array}{l}\text { Produzieren- } \\
\text { des Gewerbe }\end{array}$} & \multicolumn{2}{|c|}{$\begin{array}{l}\text { Dienst- } \\
\text { leistungen }\end{array}$} \\
\hline & $\mathrm{ABL}$ & NBL & $A B L$ & NBL & $A B L$ & NBL & $\mathrm{ABL}$ & NBL & $A B L$ & NBL & $A B L$ & NBL & $A B L$ & NBL \\
\hline 1991 & -- & -- & 7,8 & 30,0 & 5,9 & 3,5 & 68,2 & 83,5 & 2,7 & 5,0 & 35,5 & 39,0 & 61,8 & 55,9 \\
\hline 1992 & -- & -- & 6,9 & 35,8 & 5,3 & 4,8 & 70,0 & 78,2 & 2,6 & 3,8 & 34,8 & 34,9 & 62,6 & 61,3 \\
\hline 1993 & -- & -- & 4,5 & 36,5 & 4,9 & 4,5 & 70,6 & 77,8 & 2,5 & 3,3 & 33,6 & 34,5 & 63,9 & 62,2 \\
\hline 1994 & -- & -- & 4,0 & 39,4 & 5,0 & 4,6 & 69,9 & 76,6 & 2,4 & 3,1 & 32,5 & 34,6 & 65,0 & 62,3 \\
\hline 1995 & 100,0 & 100,0 & 3,5 & 36,0 & 5,1 & 3,9 & 69,7 & 78,0 & 2,3 & 3,0 & 32,0 & 34,6 & 65,8 & 62,5 \\
\hline 1996 & 100,1 & 102,0 & 3,1 & 31,0 & 5,1 & 3,8 & 69,4 & 77,2 & 2,1 & 2,8 & 31,1 & 33,5 & 66,7 & 63,7 \\
\hline 1997 & 100,3 & 103,4 & 2,9 & 27,3 & 5,4 & 3,8 & 68,6 & 75,9 & 2,0 & 2,8 & 30,5 & 32,5 & 67,5 & 64,7 \\
\hline 1998 & 100,7 & 104,4 & 3,9 & 23,9 & 5,4 & 3,7 & 68,8 & 75,2 & 2,0 & 2,8 & 30,1 & 31,2 & 67,9 & 66,1 \\
\hline 1999 & 101,1 & 105,2 & 4,6 & 20,7 & 5,1 & 3,5 & 70,0 & 75,5 & 1,9 & 2,7 & 29,5 & 29,8 & 68,6 & 67,4 \\
\hline 2000 & 101,6 & 105,8 & 5,0 & 17,1 & 4,8 & 3,4 & 71,4 & 74,9 & 1,8 & 2,7 & 28,9 & 28,7 & 69,2 & 68,6 \\
\hline 2001 & 102,3 & 106,4 & 4,3 & 10,1 & 5,1 & 4,0 & 70,7 & 72,3 & 1,8 & 2,5 & 28,7 & 27,7 & 69,6 & 69,8 \\
\hline 2002 & 103,0 & 107,0 & 2,9 & 5,9 & 5,0 & 4,0 & 70,8 & 71,6 & 1,7 & 2,5 & 28,0 & 26,6 & 70,2 & 70,9 \\
\hline 2003 & 103,7 & 107,8 & 2,4 & 5,0 & 5,0 & 4,0 & 70,7 & 71,0 & 1,7 & 2,5 & 27,5 & 26,0 & 70,8 & 71,5 \\
\hline 2004 & 104,6 & 108,5 & 2,0 & 4,5 & 6,0 & 4,8 & 67,5 & 67,6 & 1,7 & 2,4 & 26,9 & 25,5 & 71,4 & 72,1 \\
\hline 2005 & 105,4 & 109,4 & 1,9 & 3,5 & 6,3 & 4,7 & 66,3 & 67,2 & 1,7 & 2,3 & 26,4 & 25,0 & 72,0 & 72,7 \\
\hline 2006 & 106,2 & 110,3 & 3,0 & 3,7 & 7,1 & 5,3 & 63,9 & 64,9 & 1,6 & 2,2 & 25,9 & 24,7 & 72,4 & 73,1 \\
\hline 2007 & 106,9 & 111,3 & 3,3 & 4,1 & 7,2 & 5,5 & 63,2 & 63,8 & 1,6 & 2,2 & 25,8 & 24,9 & 72,5 & 72,9 \\
\hline 2008 & 107,5 & 112,3 & 3,2 & 3,6 & 6,5 & 5,2 & 65,1 & 64,9 & 1,6 & 2,2 & 25,9 & 25,1 & 72,5 & 72,7 \\
\hline 2009 & 108,0 & 113,3 & 0,9 & 0,9 & 5,3 & 4,5 & 68,2 & 67,1 & 1,6 & 2,2 & 25,4 & 24,9 & 73,0 & 72,9 \\
\hline 2010 & 108,6 & 114,5 & 1,7 & 1,7 & 6,0 & 4,9 & 66,4 & 66,1 & 1,6 & 2,2 & 25,0 & 24,8 & 73,4 & 73,0 \\
\hline 2011 & 109,2 & 115,7 & 2,9 & 2,0 & 6,3 & 5,2 & 65,7 & 64,9 & 1,6 & 2,2 & 25,1 & 25,4 & 73,3 & 72,4 \\
\hline 2012 & 109,8 & 117,1 & 2,3 & 1,9 & 5,8 & 4,9 & 67,4 & 66,2 & 1,6 & 2,2 & 25,2 & 25,6 & 73,2 & 72,2 \\
\hline 2013 & 110,5 & 118,5 & 2,0 & 0,4 & 5,7 & 5,0 & 67,8 & 66,0 & 1,5 & 2,2 & 25,2 & 25,6 & 73,4 & 72,3 \\
\hline 2014 & 111,3 & 120,1 & 2,5 & 0,6 & 5,8 & 5,1 & 67,7 & 65,8 & 1,5 & 2,2 & 25,1 & 25,6 & 73,4 & 72,2 \\
\hline 2015 & 112,2 & 121,8 & 2,5 & 0,0 & 6,0 & 5,2 & 67,4 & 66,1 & 1,4 & 2,1 & 24,9 & 25,4 & 73,7 & 72,4 \\
\hline 2016 & 113,1 & 123,7 & 2,7 & 1,5 & 6,1 & 5,2 & 67,4 & 66,4 & 1,4 & 2,1 & 24,7 & 25,2 & 73,9 & 72,7 \\
\hline 2017 & -- & -- & -- & -- & -- & -- & 67,6 & 66,7 & 1,4 & 2,1 & 24,7 & 25,1 & 74,0 & 72,8 \\
\hline 2018 & -- & -- & -- & -- & -- & -- & -- & -- & 1,3 & 2,0 & 24,8 & 25,3 & 73,9 & 72,7 \\
\hline
\end{tabular}

Anmerkung: Totale Faktorproduktivität: Kettenindex; Nettoinvestitionsquote = Nettoinvestition/BIP in \%; Rendite = Vermögenseinkommen je Nettoanlagevermögen in \%; Anteil der Arbeitsentgelte am Volkseinkommen in \%; Landwirtschaft = Prozentualer Anteil des Sektors A (Land- und Forstwirtschaft, Fischerei) an der Gesamtzahl der Erwerbstätigen im jeweiligen Gebiet; Produzierendes Gewerbe = wie oben, bezogen auf die Sektoren B-F; Dienstleistungen = wie oben, bezogen auf die Sektoren G-T. ABL: Alte Bundesländer; NBL: Neue Bundesländer.

Quelle: Volkswirtschaftliche Gesamtrechnungen der Länder; eigene Rechnungen.

werbstätigen im Sektor A sind dort also gut untergebracht - sie korrigieren die unterdurchschnittliche Produktivität ein wenig nach oben. Die Zahlen (vgl. Tabelle 1, Spalten Landwirtschaft bis Dienstleistungen) zeigen, dass sektoral gesehen eine starke Konvergenz zwischen den beiden Teilen der deutschen Volkswirtschaft existiert. Diese strukturellen Unterschiede können somit den Produktivitätsabstand (vgl. Abbildung 2) nicht erklären. ${ }^{16}$ Auch die These, dass die geringe Kapitalintensität im Osten auf das Vor-

16 Wenn H. Beitz et al. behaupten, dass „strukturelle“ „Einflüsse zum großen Teil das geringe Produktivitätsniveau in Ostdeutschland“ erklären, so müssten sie deutlicher sagen, welche sie meinen. Eine stärkere ländliche Prägung kommt jedenfalls nicht infrage, denn dann wäre das Produktivitätsniveau höher. herrschen der Dienstleistungen, in denen weniger Kapital gebraucht wird, zurückzuführen sei, widerspricht den Fakten, da der westdeutsche Dienstleistungssektor prozentual und im Durchschnitt stärker ausgeprägt ist.

\section{Ausstattung eines Arbeitsplatzes mit moderner Technik}

Volkswirtschaftlich gesehen ist eine Erklärung der Produktivitätsunterschiede mithilfe der durchschnittlichen Ausstattung eines Arbeitsplatzes mit moderner Technik, die durch die Kapitalintensität gemessen wird, plausibel: Schließlich müssen Arbeit und Kapital zusammenwirken, um eine hohe Produktivität zu erreichen. Zunächst ist festzuhalten, dass das produktive Kapital, gemessen am realen Nettoanlage- 
vermögen, in beiden Teilen Deutschlands wächst. Die neuen Bundesländer erlebten ein besonders starkes Wachstum bis 2000, danach erhöhte sich der Kapitalstock im Unterschied zu den alten Bundesländern eher verhalten. Trotzdem sinkt seit einigen Jahren die Ausstattung eines Arbeitsplatzes mit Investitionsgütern sowohl im Osten als auch im Westen. Während dieser Effekt in den alten Bundesländern vor allem durch die wachsende Zahl an Erwerbstätigen verursacht wird, ist diese Zahl in den neuen Bundesländern nahezu konstant geblieben. Die Ursache muss deshalb anderweitig gesucht werden, und man findet sie in der sinkenden Nettoinvestitionsquote im Osten, die 2015 bereits einmal auf null gesunken war (vgl. Tabelle 1). ${ }^{17}$ Die Produktivitätszuwächse in den neuen Bundesländern werden trotz einer deutlich geringeren und vor allem stagnierenden Kapitalausstattung je Arbeitsplatz allein aufgrund des technologischen Fortschrittes erzielt, der auf der Einführung modernster Technik im Zuge der Ersatzinvestitionen basiert. Ergänzend wäre festzuhalten, dass die Vermögenseinkommen die Nettoinvestitionen seit 2000 bei weitem übersteigen; höhere Investitionen wären also durchaus finanzierbar. ${ }^{18}$

Gegen die hier vertretene These, dass die geringere Produktivität im Osten durch die geringere Kapitalintensität erklärt werden kann, wird manchmal geltend gemacht, dass bei ähnlicher Kapitalintensität durchaus unterschiedliche Faktorproduktivitäten beobachtet werden können. Das ist nicht zu bestreiten, ist aber kein Gegenargument. Die Produktivität hängt bekanntlich nicht nur von der Kapitalintensität ab, sondern auch von der zugrunde liegenden Technologie. So kann ein- und dieselbe Menge Kapital in ganz unterschiedlicher Technik investiert worden sein. Aus diesem Grund ist es erforderlich, den „neutralen technologischen Fortschritt“ in die Analyse einzu-

17 Bedingt durch hohe Abschreibungsraten ist die in der offiziellen Statistik berichtete Bruttoinvestitionsquote der neuen Bundesländer meistens höher als in den alten Ländern. Das Defizit an Erweiterungsinvestitionen, die für die Konvergenz bei der Produktivität wichtig sind, wird erst sichtbar, wenn man die Nettoinvestitionsquote heranzieht.

18 Das Vermögenseinkommen wird berechnet, indem vom Einkommen aus Unternehmertätigkeit und Vermögen der sogenannte Unternehmerlohn abgezogen wird. Dieser ergibt sich aus den durchschnittlichen Arbeitsentgelten je Arbeitnehmer multipliziert mit der Zahl der Selbständigen. beziehen, ${ }^{19}$ zumindest, wenn die Datenverfügbarkeit das hergibt.

Die geringe Neigung, trotz steigender Vermögenseinkommen im Osten zu investieren, könnte etwas mit dem Verhältnis dieser Einkommen zum Kapitaleinsatz zu tun haben. Ein Maß dafür ist die Rendite, hier berechnet mit Bezug auf das jeweilige nominale Nettoanlagevermögen (vgl. Tabelle 1). Sie liegt im Osten im Durchschnitt mindestens 1 Prozentpunkt unter dem Westniveau, wobei allerdings starke Schwankungen auftreten. Diese Differenz wäre noch größer, wenn sich Löhne und Gehälter stärker dem Westniveau angepasst hätten. Trotz der geringeren Profitabilität ist der Anteil der Arbeitsentgelte am Volkseinkommen im Osten niedriger als im Westen (vgl. Tabelle 1); wäre er höher, würden die Profite sinken. Fakt ist auch: In beiden Teilen Deutschlands nimmt der Anteil der Arbeitsentgelte am Volkseinkommen seit 1991 ziemlich stetig ab.

\section{Zusammenfassung}

Das dynamische Gleichgewicht, das sich in der deutschen Volkswirtschaft herausgebildet hat, ist überwiegend durch eine Annäherung der neuen Bundesländer an die Struktur und die Parameter der alten Bundesländer charakterisiert. Im Osten entwickelte sich die Produktivität besonders dynamisch und in den letzten Jahren fast ausschließlich aufgrund des technischen Fortschritts. Dass eine Steigerung der Kapitalintensität den Konvergenzprozess unterstützen würde, kann wohl kaum bestritten werden. Wenn eine inzwischen profitabel gewordene Wirtschaft wachsen soll, müssen die Gewinne dort investiert werden, wo sie entstehen, ganz gleich, ob auf dem Land oder in der Stadt. Dazu wären stärkere Anreize für unternehmerische Investitionen ein geeignetes Mittel. Angesichts der Größenordnung zusätzlich zu investierender Beträge - es fehlen ca. 45 Mrd. Euro, um die Kapitalausstattung anzugleichen - dürfte der Staat sehr schnell überfordert sein, wollte man ihm allein diese Aufgabe zuweisen.

19 R. M. Solow: Technical Change and the Aggregate Production Function, in: The Review of Economics and Statistics, 39. Jg. (1957), H. 3, S. 312-320.

Title: The East is Catching Up, But Needs More Investment

Abstract: The productive factors (number of employed persons and fixed assets) are 6.5 times more pronounced in the west of Germany than in the east; however, the performance, measured against the real gross domestic product, is 7.7 times higher. The difference is the result of higher productivity in manufacturing and services. Although agriculture is comparatively more productive in the new federal states, this is of little significance given the $2 \%$ share of the sectoral structure. From a dynamic perspective, the productivity comparison shows that Eastern Germany has been catching up since 2011 after a phase of stagnation.

JEL Classification: O47, L16, R12 